\title{
Topology optimization of structures: a minimum weight approach with stress constraints
}

\author{
F. Navarrina*, I. Muiños, I. Colominas, M. Casteleiro \\ GMNI - Grupo de Métodos Numéricos en Ingeniería, \\ Department of Applied Mathematics, Universidad de A Coruña, \\ E.T.S. de Ingenieros de Caminos, Canales y Puertos \\ Campus de Elviña, 15192 A Coruña, SPAIN
}

\begin{abstract}
Sizing and shape structural optimization problems are normally stated in terms of a minimum weight approach with constraints that limit the maximum allowable stresses and displacements.

However, topology structural optimization problems have been usually stated in terms of a maximum stiffness (minimum compliance) approach. In this kind of formulations, the aim is to distribute a given amount of material in a certain domain, so that the stiffness of the resulting structure is maximized (the compliance, or energy of deformation, is minimized) for a given load case. Thus, the material mass is restricted to a predefined percentage of the maximum possible mass, while no stress or displacement constraints are taken into account.

In this paper we analyze and compare both approaches, and we present a FEM minimum weight with stress constraints (MWSC) formulation for topology structural optimization problems. This approach does not require any stabilization technique to produce acceptable optimized results, while no truss-like final solutions are necessarily obtained. Several 2D examples are presented. The optimized solutions seem to be correct from the engineering point of view, and their appearence could be considered closer to the engineering intuition than the traditional truss-like results obtained by means of the widespread maximum stiffness (minimum compliance) approaches.
\end{abstract}

Key words: Structural optimization, Topology optimization, Optimum design PACS: 02.60.PN, 02.70.Dh, 46.15.-x

* Corresponding author.

Email address: fnavarrina@udc.es (F. Navarrina).

URL: http://caminos.udc.es/gmni. 


\section{Introduction}

Around four decades ago Schmidt [1] proposed a revolutionary idea that gave rise to a new discipline: engineers, as a general rule, try to design minimum cost objects or systems that must withstand the maximum unfavorable estimated loads; therefore, (optimum) design could be systematically stated in terms of constrained minimization problems; then, these problems could be solved by means of mathematical programming techniques implemented in high speed digital computers. Sizing and shape structural optimization problems have been thereafter mainly written in terms of minimum weight formulations, with non linear constraints that limit the maximum allowable stresses and displacements $[2,3,4]$.

However, since Bendse and Kikuchi proposed the basic concepts [5] in 1988, most of topology structural optimization problems have been routinely stated in terms of minimum compliance (maximum stiffness) approaches. In this kind of formulations, one tries to distribute a given amount of material within a given domain, so that the stiffness of the resulting structure is maximized (the compliance is minimized) for a given load case [6]. The traditional minimum compliance formulations offer some obvious advantages, since one avoids dealing with a large number of highly non-linear constraints. This could be considered crucial, if one takes into account the large number of design variables that is inherent to topology optimization. However, one can also argue that this gives rise to several important drawbacks. Thus, multiple load cases can not be considered, different solutions are obtained for different restrictions on the amount of material, and the final design could be unfeasible in practice, since no constraints are imposed on stresses and displacements. Moreover, the minimum compliance problem is said to be ill-posed, since the solution oscillates as the discretization refinement is increased $[7,8,9]$, although this difficulty can be partially overcome by introducing porous materials.

The SIMP (solid isotropic material with penalty) formulation $[7,9,10]$ is the most widely used minimum compliance approach, so far. In this formulation, one introduces a non-dimensional design variable per element (the relative density, that is the one's complement of the porosity), which value ranges from 0 to 1 . The aim is to compute the design variables (the amount of porous material that must be distributed within each element) in such a way that a highly non-linear objective function is minimized (the compliance, or energy of deformation), while a single linear constraint is satisfied (the total amount of material is limited, by the so called filling factor, to occupy a predefined percentage of the domain volume). The SIMP formulation is easy to implement in a FEM framework. Moreover, several explicit procedures have been proposed to iterate the relative density of the elements [7,9], what facilitates solving the minimization problem. However, a spread porous material distri- 
bution is frequently considered an unwanted result, and numerical instabilities do often occur. Therefore, additional penalization and stabilization techniques must still be employed $[7,9]$. On the other hand, an image filter is generally applied, in order to enable the final interpretation of the results. These normally resemble truss-like structures [7].

But one could question these results, since the final design depends on so many arbitrary parameters (filling factor, degree of discretization, applied penalization and stabilization techniques, image filter, etc.) One could even say that the final results are somehow impelled to resemble truss-like structures, since this is what one expects to obtain as a satisfactory final design. On the other hand, the final design could be unfeasible in practice, since the formulation disregards the stress and displacement fields. And, obviously, the stiffest design that can be built with a given amount of material will normally differ from the cheapest (in cost of material) design that can support a set of given load cases.

Next, we present a FEM minimum weight with stress constraints (MWSC) approach for topology structural optimization problems $[9,11,12]$.

\section{The Structural Analysis Model}

\subsection{The Structural Analysis Problem}

Let $\Omega^{\circ}$ be a domain in the material space originally occupied by a certain deforming body. Due to given external loads the body is deformed onto a different domain $\Omega$. Thus, every arbitrary point $P^{o}$ in $\Omega^{o}$ is carried into a different position $P$ in $\Omega$. Let $\boldsymbol{r}^{o}$ and $\boldsymbol{r}$ be the material coordinates vectors of points $P^{o}$ and $P$, respectively. Our aim is to compute the displacements

$$
\boldsymbol{u}\left(\boldsymbol{r}^{o}\right)=\boldsymbol{r}\left(\boldsymbol{r}^{o}\right)-\boldsymbol{r}^{o}
$$

which are the key to obtain the strains $\boldsymbol{\varepsilon}\left(\boldsymbol{r}^{o}\right)$ and the stresses $\boldsymbol{\sigma}\left(\boldsymbol{r}^{o}\right)$. In linear elasticity with small displacements and small displacement gradients the corresponding expressions are

$$
\varepsilon=L u, \quad \sigma=D \varepsilon
$$

Let the external loads be the forces $\boldsymbol{b}\left(\boldsymbol{r}^{o}\right)$ per unit volume (of the body) in the domain $\Omega^{o}$, and $\boldsymbol{t}\left(\boldsymbol{r}^{o}\right)$ per unit area on the surface $\Gamma_{\sigma}^{o}$. In these terms, the 
structural analysis problem can be written as $[13,14]$

$$
\begin{array}{ll}
\text { Find } & \boldsymbol{u} \in H_{u} \\
\text { such that } & a(\boldsymbol{w}, \boldsymbol{u})=(\boldsymbol{w}, \boldsymbol{b})_{\Omega^{o}}+(\boldsymbol{w}, \boldsymbol{t})_{\Gamma_{\sigma}^{o}} \quad \forall \boldsymbol{w} \in H_{w} \\
\text { being } & a(\boldsymbol{w}, \boldsymbol{u})=\iiint_{\Omega^{o}}(\boldsymbol{L} \boldsymbol{w})^{T} \boldsymbol{D}(\boldsymbol{L u}) d \Omega, \\
& (\boldsymbol{w}, \boldsymbol{b})_{\Omega^{o}}=\iiint_{\Omega^{o}} \boldsymbol{w}^{T} \boldsymbol{b} d \Omega, \quad(\boldsymbol{w}, \boldsymbol{t})_{\Gamma_{\sigma}^{o}}=\iint_{\Gamma_{\sigma}^{o}} \boldsymbol{w}^{T} \boldsymbol{t} d \Gamma,
\end{array}
$$

where the trial functions $\boldsymbol{u}$ and the test functions $\boldsymbol{w}$ are required to satisfy the essential boundary conditions (prescribed displacements) and their corresponding homogeneous boundary conditions, respectively.

\subsection{The Finite Element Numerical Model}

As a general rule, it will not be possible to obtain the exact solution of the above stated problem. Hence, we will try to approximate the exact solution in a finite-dimensional context. Thus, we replace the function spaces $H_{u}$ and $H_{w}$ by their respective finite dimension subspaces $H_{u}^{h}$ and $H_{w}^{h}$. Let $\boldsymbol{u}^{h}$ and $\boldsymbol{w}^{h}$ be the discretized trial and test functions in the above mentioned subespaces. Let $\boldsymbol{u}^{p}$ be a trial function that satisfies the essential boundary conditions. And let $\left\{\phi_{i}\left(\boldsymbol{r}^{o}\right)\right\}$ and $\left\{w_{j}\left(\boldsymbol{r}^{o}\right)\right\}$ be conveniently selected bases of discretized trial and test functions in the corresponding subespaces $H_{u}^{h}$ and $H_{w}^{h}$, verifying the homogeneous boundary conditions of our problem. In this terms we can write $[13,14]$

$$
\begin{array}{rlrl}
\boldsymbol{u}^{h}\left(\boldsymbol{r}^{o}\right) & =\boldsymbol{u}^{p}\left(\boldsymbol{r}^{o}\right)+\sum_{i=1}^{N} \boldsymbol{\Phi}_{i}\left(\boldsymbol{r}^{o}\right) \boldsymbol{\alpha}_{i}, & & \boldsymbol{\Phi}_{i}\left(\boldsymbol{r}^{o}\right)=\phi_{i}\left(\boldsymbol{r}^{o}\right) \boldsymbol{I}_{3}, \\
\boldsymbol{w}^{h}\left(\boldsymbol{r}^{o}\right) & =\sum_{j=1}^{N} \boldsymbol{W}_{j}\left(\boldsymbol{r}^{o}\right) \boldsymbol{\beta}_{j}, & \boldsymbol{W}_{j}\left(\boldsymbol{r}^{o}\right)=w_{j}\left(\boldsymbol{r}^{o}\right) \boldsymbol{I}_{3} .
\end{array}
$$

In a FEM formulation the unknown $\boldsymbol{\alpha}_{i}$ is the nodal displacements vector of node number $i$. Moreover, the domain $\Omega^{\circ}$ is discretized in such a way that

$$
\bar{\Omega}^{o}=\bigcup_{e=1}^{\text {nelem }} \bar{E}_{e}, \quad E_{e_{1}} \bigcap E_{e_{2}}=\emptyset \quad \forall e_{1} \neq e_{2},
$$

being $E_{e}$ the so-called finite elements. On the other hand, in solid mechanics one normally resorts to a Galerkin type formulation, by taking the same base for both, the trial and the test functions. Thus,

$$
w_{j}\left(\boldsymbol{r}^{o}\right)=\phi_{j}\left(\boldsymbol{r}^{o}\right)
$$


Therefore, the FEM numerical model of structural analysis can be written as

$$
\begin{array}{ll}
\text { Find } & \boldsymbol{\alpha}=\left\{\boldsymbol{\alpha}_{i}\right\}, \quad i=1, \ldots, N \\
\text { such that } & \sum_{i=1}^{N} \boldsymbol{K}_{j i} \boldsymbol{\alpha}_{i}=\boldsymbol{f}_{j}, \quad j=1, \ldots, N \\
\text { being } & \boldsymbol{K}_{j i}=a\left(\boldsymbol{\Phi}_{j}, \boldsymbol{\Phi}_{i}\right), \\
& \boldsymbol{f}_{j}=\left(\boldsymbol{\Phi}_{j}, \boldsymbol{b}\right)_{\Omega^{o}}+\left(\boldsymbol{\Phi}_{j}, \boldsymbol{t}\right)_{\Gamma_{\sigma}^{o}}-a\left(\boldsymbol{\Phi}_{j}, \boldsymbol{u}^{p}\right) .
\end{array}
$$

The required terms can be computed on an element by element sequence. Thus,

$$
\begin{aligned}
& \boldsymbol{K}_{j i}=\sum_{e=1}^{n e l e m} \boldsymbol{K}_{j i}^{e}, \\
& \boldsymbol{f}_{j}=\iint_{\Gamma_{\sigma}^{o}} \boldsymbol{\Phi}_{j}^{T} \boldsymbol{t} d \Gamma+\sum_{e=1}^{\text {nelem }} \boldsymbol{f}_{j}^{e},
\end{aligned}
$$

being the element contributions

$$
\begin{aligned}
& \boldsymbol{K}_{j i}^{e}=\iiint_{E_{e}}\left(\boldsymbol{L} \boldsymbol{\Phi}_{j}\right)^{T} \boldsymbol{D}\left(\boldsymbol{L} \boldsymbol{\Phi}_{i}\right) d \Omega \\
& \boldsymbol{f}_{j}^{e}=\iiint_{E_{e}}\left(\boldsymbol{\Phi}_{j}^{T} \boldsymbol{b}-\left(\boldsymbol{L} \boldsymbol{\Phi}_{j}\right)^{T} \boldsymbol{D}\left(\boldsymbol{L} \boldsymbol{u}^{p}\right)\right) d \Omega .
\end{aligned}
$$

Once the solution $\boldsymbol{\alpha}$ to problem (7) is found, we can compute at any point $\boldsymbol{r}^{o} \in \Omega^{o}$ the aproximations

$$
\begin{gathered}
\boldsymbol{u}^{h}\left(\boldsymbol{r}^{o}\right)=\boldsymbol{u}^{p}\left(\boldsymbol{r}^{o}\right)+\sum_{i=1}^{N} \boldsymbol{\Phi}_{i}\left(\boldsymbol{r}^{o}\right) \boldsymbol{\alpha}_{i} \\
\boldsymbol{\varepsilon}^{h}\left(\boldsymbol{r}^{o}\right)=\boldsymbol{L} \boldsymbol{u}^{h}\left(\boldsymbol{r}^{o}\right), \quad \boldsymbol{\sigma}^{h}\left(\boldsymbol{r}^{o}\right)=\boldsymbol{D} \boldsymbol{\varepsilon}^{h}\left(\boldsymbol{r}^{o}\right) .
\end{gathered}
$$

\section{MWSC Formulation}

\subsection{The Structural Analysis Problem with Relative Density}

Let the domain $\Omega^{o}$ be occupied by a porous material. Let $\rho\left(\boldsymbol{r}^{o}\right)$ be the relative density of the material (one's complement of the porosity, which adimensional value must range from 0 to 1 ) at point $\boldsymbol{P}^{o}$ of material coordinates $\boldsymbol{r}^{o}$. For a given distribution of (porous) material, defined by the relative density field $\rho\left(\boldsymbol{r}^{o}\right)$, our aim is to compute the displacements (1) and the associated strains and stresses (2). We assume again the linear elasticity hypothesis, what implies small displacements and small displacement gradients. 
Let $d \Omega$ be the volume of a differential region in the vicinity of point $P^{o}$. By definition, the volume occupied by the porous material within the differential region will be $\rho\left(\boldsymbol{r}^{o}\right) d \Omega$. Therefore, the structural analysis problem (3) can be written as $[9]$

$$
\begin{aligned}
& \text { Given } \quad \rho\left(\Omega^{o}\right) \\
& \text { find } \quad \boldsymbol{u} \in H_{u} \\
& \text { such that } a(\boldsymbol{w}, \boldsymbol{u})=(\boldsymbol{w}, \boldsymbol{b})_{\Omega^{o}}+(\boldsymbol{w}, \boldsymbol{t})_{\Gamma_{\sigma}^{o}} \quad \forall \boldsymbol{w} \in H_{w} \\
& \text { being } \quad a(\boldsymbol{w}, \boldsymbol{u})=\iiint_{\Omega^{o}}(\boldsymbol{L} \boldsymbol{w})^{T} \boldsymbol{D}(\boldsymbol{L} \boldsymbol{u}) \rho d \Omega \text {, } \\
& (\boldsymbol{w}, \boldsymbol{b})_{\Omega^{o}}=\iiint_{\Omega^{o}} \boldsymbol{w}^{T} \boldsymbol{b} \rho d \Omega, \quad(\boldsymbol{w}, \boldsymbol{t})_{\Gamma_{\sigma}^{o}}=\iint_{\Gamma_{\sigma}^{o}} \boldsymbol{w}^{T} \boldsymbol{t} d \Gamma .
\end{aligned}
$$

Notice that, in comparison with the original statement (3), the modifications are reduced to taking into account the porosity effect in the integration. In fact, once the displacements are known, the strain and stress fields are computed with the same expressions, independently of the actual material distribution. However, we must exclude the case in which the relative densitity is locally null, since the concepts of displacement, strain and stress become meaningless.

It is worthy to reflect on the physical meaning of the stress $\boldsymbol{\sigma}\left(\boldsymbol{r}^{o}\right)$ computed by means of expressions (2). It really represents the stress tensor of the deforming body. However, we recall that in the vicinity of each point there are probably regions occupied by material, as much as empty ones. Therefore, if we try to analyze the forces equilibrium in a finite subdomain, we should operate with the so-called effective stress, by multiplying the stress $\boldsymbol{\sigma}\left(\boldsymbol{r}^{o}\right)$ by the relative density $\rho\left(\boldsymbol{r}^{o}\right)$.

\subsection{The Finite Element Numerical Model with Relative Density}

Let $\rho_{e}$ be the relative density of element number $e$, what is assumed constant within the element. Let $\boldsymbol{\rho}=\left\{\rho_{e}\right\}(e=1, \ldots$, nelem $)$ be the relative densities vector, that will constitute the design variables of the topology optimization problem. For a given $\boldsymbol{\rho}$, the structural analysis problem to be solved is:

$$
\begin{array}{ll}
\text { Find } & \boldsymbol{\alpha}(\boldsymbol{\rho}) \\
\text { such that } & \sum_{i=1}^{N} \boldsymbol{K}_{j i}(\boldsymbol{\rho}) \boldsymbol{\alpha}_{i}(\boldsymbol{\rho})=\boldsymbol{f}_{j}(\boldsymbol{\rho}), \quad j=1, \ldots, N,
\end{array}
$$


The required terms can be computed on an element by element sequence. Thus

$$
\begin{aligned}
& \boldsymbol{K}_{j i}(\boldsymbol{\rho})=\sum_{e=1}^{\text {nelem }} \boldsymbol{K}_{j i}^{e}\left(\rho_{e}\right), \\
& \boldsymbol{f}_{j}(\boldsymbol{\rho})=\iint_{\Gamma_{\sigma}^{o}} \boldsymbol{\Phi}_{j}^{T} \boldsymbol{t} d \Gamma+\sum_{e=1}^{n e l e m} \boldsymbol{f}_{j}^{e}\left(\rho_{e}\right),
\end{aligned}
$$

being the element contributions

$$
\begin{aligned}
& \boldsymbol{K}_{j i}^{e}\left(\rho_{e}\right)=\iiint_{E_{e}}\left(\boldsymbol{L} \boldsymbol{\Phi}_{j}\right)^{T} \boldsymbol{D}\left(\boldsymbol{L} \boldsymbol{\Phi}_{i}\right) \rho_{e} d \Omega \\
& \boldsymbol{f}_{j}^{e}\left(\rho_{e}\right)=\iiint_{E_{e}}\left(\boldsymbol{\Phi}_{j}^{T} \boldsymbol{b}-\left(\boldsymbol{L} \boldsymbol{\Phi}_{j}\right)^{T} \boldsymbol{D}\left(\boldsymbol{L} \boldsymbol{u}^{p}\right)\right) \rho_{e} d \Omega .
\end{aligned}
$$

Once the solution $\boldsymbol{\alpha}(\boldsymbol{\rho})$ to problem (13) is found, we can compute at any arbitrary point $\boldsymbol{r}^{o} \in \Omega^{o}$ the aproximations

$$
\begin{gathered}
\boldsymbol{u}^{h}\left(\boldsymbol{r}^{o}, \boldsymbol{\rho}\right)=\boldsymbol{u}^{p}\left(\boldsymbol{r}^{o}\right)+\sum_{i=1}^{N} \boldsymbol{\Phi}_{i}\left(\boldsymbol{r}^{o}\right) \boldsymbol{\alpha}_{i}(\boldsymbol{\rho}) \\
\boldsymbol{\varepsilon}^{h}\left(\boldsymbol{r}^{o}, \boldsymbol{\rho}\right)=\boldsymbol{L} \boldsymbol{u}^{h}\left(\boldsymbol{r}^{o}, \boldsymbol{\rho}\right), \quad \boldsymbol{\sigma}^{h}\left(\boldsymbol{r}^{o}, \boldsymbol{\rho}\right)=\boldsymbol{D} \boldsymbol{\varepsilon}^{h}\left(\boldsymbol{r}^{o}, \boldsymbol{\rho}\right) .
\end{gathered}
$$

By comparing (16) and (17) with (10) and (11) we observe that displacements, strains and stresses are still computed in the usual way. Therefore, if we wish to adapt an existing FEM numerical model of structural analysis as a component of a topology optimization system, we only have to modify the element contributions (9) computation. Moreover, the required adjustment is quite simple, since we only need to introduce the relative density in the integration of the corresponding expressions (15). Furthermore, computing contributions (15) is fairly straightforward, since we assume that the relative density is constant within each element. Thus, we just have to multiply the original results (9) by the corresponding relative densities. On the other hand, the original results (9) give the first order derivatives of contributions (15) with respect to the design variables. Moreover, all the other first and higher order derivatives are obviously null.

We conclude that we do not have to modify the source at the lower level for adapting an existing FEM code into a topology optimization system. In practice, only slight adjustments must be implemented in the data flow between the higher level routines. In fact, any conventional code should contain all the basic tools to perform the required new computations and the associated sensitivity analysis. 


\subsection{Statement of the Stress Constraints}

The values $\boldsymbol{\sigma}^{h}\left(\boldsymbol{r}^{o}, \boldsymbol{\rho}\right)$ computed by means of (16) and (17) are numerical approximations to the actual stress tensor components of the material being deformed. Thus, the allowable values of the reference stress $\widehat{\sigma}(\boldsymbol{\sigma})$ at point $\boldsymbol{r}_{j}^{o}$ can be limited by introducing constraints type

$$
\begin{aligned}
& g_{j}(\boldsymbol{\rho})=\widehat{\sigma}\left(\boldsymbol{\sigma}^{h}\left(\boldsymbol{r}_{j}^{o}, \boldsymbol{\rho}\right)\right)-\widehat{\sigma}_{\text {max }} \leq 0, \quad \text { or } \\
& g_{j}(\boldsymbol{\rho})=\widehat{\sigma}_{\text {min }}-\widehat{\sigma}\left(\boldsymbol{\sigma}^{h}\left(\boldsymbol{r}_{j}^{o}, \boldsymbol{\rho}\right)\right) \leq 0,
\end{aligned}
$$

where $\widehat{\sigma}_{\max }$ and $\widehat{\sigma}_{\min }$ are the corresponding upper and lower limits. However, since we are dealing with a porous material, we could state alternative expressions in terms of the effective stress. As a general rule, this is as simple as multiplying the above expressions by the relative density $\rho\left(\boldsymbol{r}_{j}^{o}\right)$, what gives

$$
\begin{aligned}
& g(\boldsymbol{\rho})=\left[\widehat{\sigma}\left(\boldsymbol{\sigma}^{h}\left(\boldsymbol{r}_{j}^{o}, \boldsymbol{\rho}\right)\right)-\widehat{\sigma}_{\text {max }}\right] \rho\left(\boldsymbol{r}_{j}^{o}\right) \leq 0, \quad \text { or } \\
& g(\boldsymbol{\rho})=\left[\widehat{\sigma}_{\text {min }}-\widehat{\sigma}\left(\boldsymbol{\sigma}^{h}\left(\boldsymbol{r}_{j}^{o}, \boldsymbol{\rho}\right)\right)\right] \rho\left(\boldsymbol{r}_{j}^{o}\right) \leq 0 .
\end{aligned}
$$

It is obvious that expressions (18) and (19) are equivalent, unless the relative density is null. This is a singular but conceptually important case, since it happens when all the material has been removed in the vicinity of the point being considered. Apparently, the difference between both ways of imposing the constraints seems to be insignificant. However, this could become a critical point, with unforeseeable effects on the performance of the optimization algorithm and the final result.

To clarify this point we resort to an academic conceptual problem. Figure 1 (left) shows the rectangular solid section of a beam with height $2 c$ and width $b$. Let $\sigma_{e}$ be the elastic stress limit of the material. The section supports the bending momment $M_{f}=2 b c^{2} \sigma_{e} / 3$, that is the maximum that can be applied without exceeding the elastic stress limit. We add an upper layer and a lower layer of porous material, both with height $\eta c(\eta<<1)$, and we keep the same value of the bending momment. In these conditions we state the following (trivial) topology optimization problem: find the relative density $\rho$ of the material in the upper and lower layers such that the weight is minimized and the elastic stress limit is not exceeded. It seems obvious that the exact solution of this problem must be $\rho=0$.

A quite simple strength of materials analysis [12] shows that the stress con- 

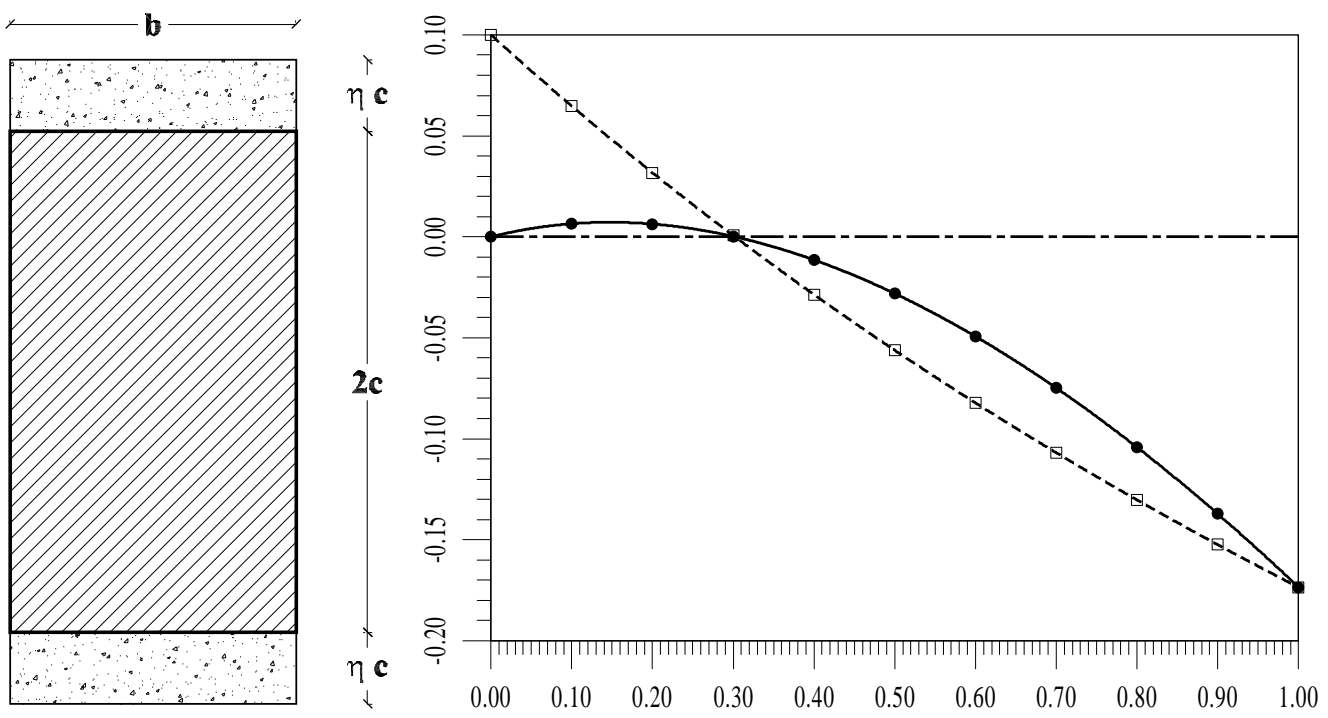

Figure 1. Layout (left) of an academic conceptual topology optimization problem,

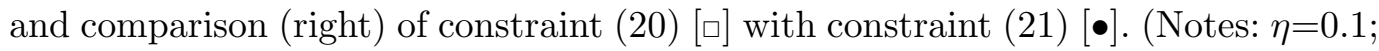
the constraint is scaled by $\sigma_{e}$.)

straint type (18) associated to this problem can be written as

$$
g(\rho)=\left[\frac{\eta-\left(3 \eta+3 \eta^{2}+\eta^{3}\right) \rho}{1+\left(3 \eta+3 \eta^{2}+\eta^{3}\right) \rho}\right] \quad \sigma_{e} \leq 0 .
$$

Figure 1 (right) shows that this constraint is not satisfied for values of the relative density under $\rho \approx 1 / 3$. Moreover, the constratint is more severely violated as we get closer to the exact solution $\rho=0$ ! It seems clear that we are facing a situation in which reaching the optimum calls for removing all the material. However, in the vicinity of the optimum (that is for any value of $\rho$ slightly greater than 0 ) the constraint is largely violated. Furthermore, its gradient is negative. This is even worse, since any consistent non linear programming algorithm will try to raise the value of the relative density, what precludes convergence to the exact solution of the problem. At the best of times we could only obtain a non global optimum.

If we rewrite constraint (20) in terms of the effective stress (that is, multiplying the above inequality by the relative density) we obtain the alternative stress constraint type (19)

$$
g(\rho)=\left[\frac{\eta-\left(3 \eta+3 \eta^{2}+\eta^{3}\right) \rho}{1+\left(3 \eta+3 \eta^{2}+\eta^{3}\right) \rho}\right] \rho \sigma_{e} \leq 0 .
$$

Figure 1 (right) shows that this constraint is still not satisfied for values of the relative density under $\rho \approx 1 / 3$. However, the reformulated constraint is strictly verified at the solution $\rho=0$. And, most important, the gradient is now positive in the vicinity of this point. Therefore, for initial values of $\rho$ not 
too far from the exact solution (less than $1 / 6$ approximately) any consistent non linear programming algorithm will try to reduce the value of the relative density, what allows to achieve convergence.

This is a critical aspect of these formulations. The challenge is to find a convenient way for limiting the stress, without overestimating the strength nor trending to fill in regions that should actually be hollowed out. The statement type (19) partially fulfills these requirements. However, it seems to slow down the converge. We have performed a few numerical tests, and this seems to be a quite promising line, although the results are not yet conclusive. A more detailed discussion on this topic can be found in [9].

\subsection{The Optimization Problem}

Let $\gamma_{\text {mat }}$ be the density of the material. We define the objective function

$$
F(\boldsymbol{\rho})=\int_{\Omega} \rho^{\frac{1}{p}} \gamma_{m a t} d \Omega=\sum_{e=1}^{\text {nelem }}\left(\rho_{e}\right)^{\frac{1}{p}} \int_{E_{e}} \gamma_{m a t} d \Omega,
$$

where $p$ is a tuning parameter that can be used to favor a mainly compact $(p>1)$ or a mainly porous $(p<1)$ distribution of material. In this terms, the topology optimization problem can be written as

$$
\begin{array}{lll}
\text { Find } & \boldsymbol{\rho}=\left\{\boldsymbol{\rho}_{e}\right\}, & e=1, \ldots, \text { nelem } \\
\text { that minimizes } & F(\boldsymbol{\rho}) & \\
\text { verifying } & g_{j}(\boldsymbol{\rho}) \leq 0, & j=1, \ldots, \text { m } \\
& 0<\rho_{\min } \leq \rho_{e} \leq 1, & e=1, \ldots, \text { nelem }
\end{array}
$$

where the stress constraints $g_{j}$ (at the corresponding points $\boldsymbol{r}_{j}^{o}$ ) must be stated accordingly to the previously exposed concepts, and the stress values $\boldsymbol{\sigma}^{h}\left(\boldsymbol{r}_{j}^{o}, \boldsymbol{\rho}\right)$ are computed by means of the proposed numerical model. Obviously, we can consider displacement constraints too. On the other hand, we introduce a lower limit for the relative density, since the entire hollowing out of some elements could cause a singular stiffness matrix and stall the optimization process. We emphasize that this topology optimization aproach is a kind of sizing optimization from the operational point of view, since the design variables do not modify the shape of the elements. The above stated formulation has been imlemented by following the general methodology [3], and applying the sensitivity analysis techniques [4] and the improved SLP algorithm with quadratic line-search [15] developed by the authors. 

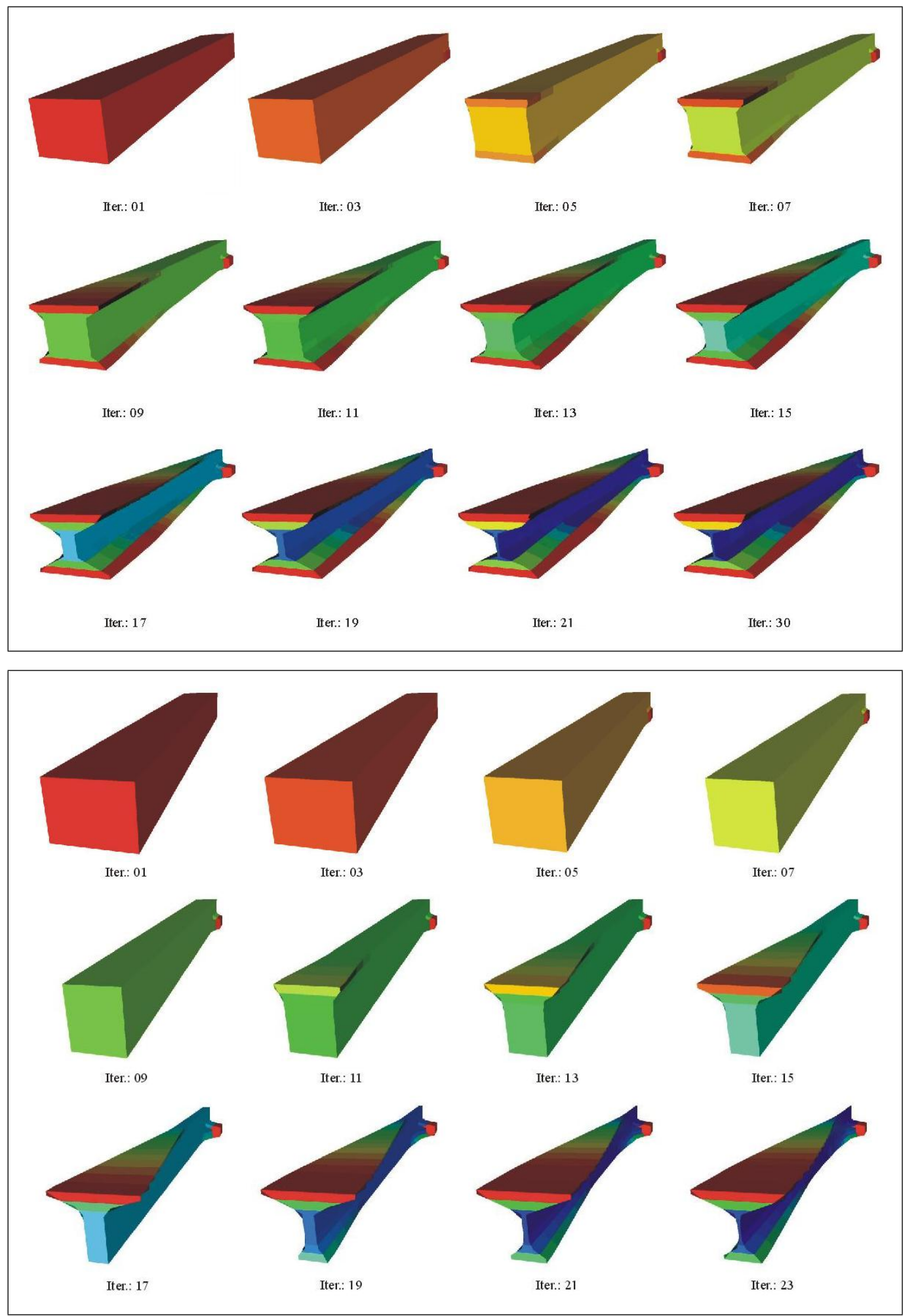

Figure 2. MWSC topology optimization of a simply supported structure, with small height/length ratio, considering sliding (up) and fixed (down) supports. Concentrated load applied in the center of the upper side. (Note: only half of the solid is represented in order to show the central section.) 

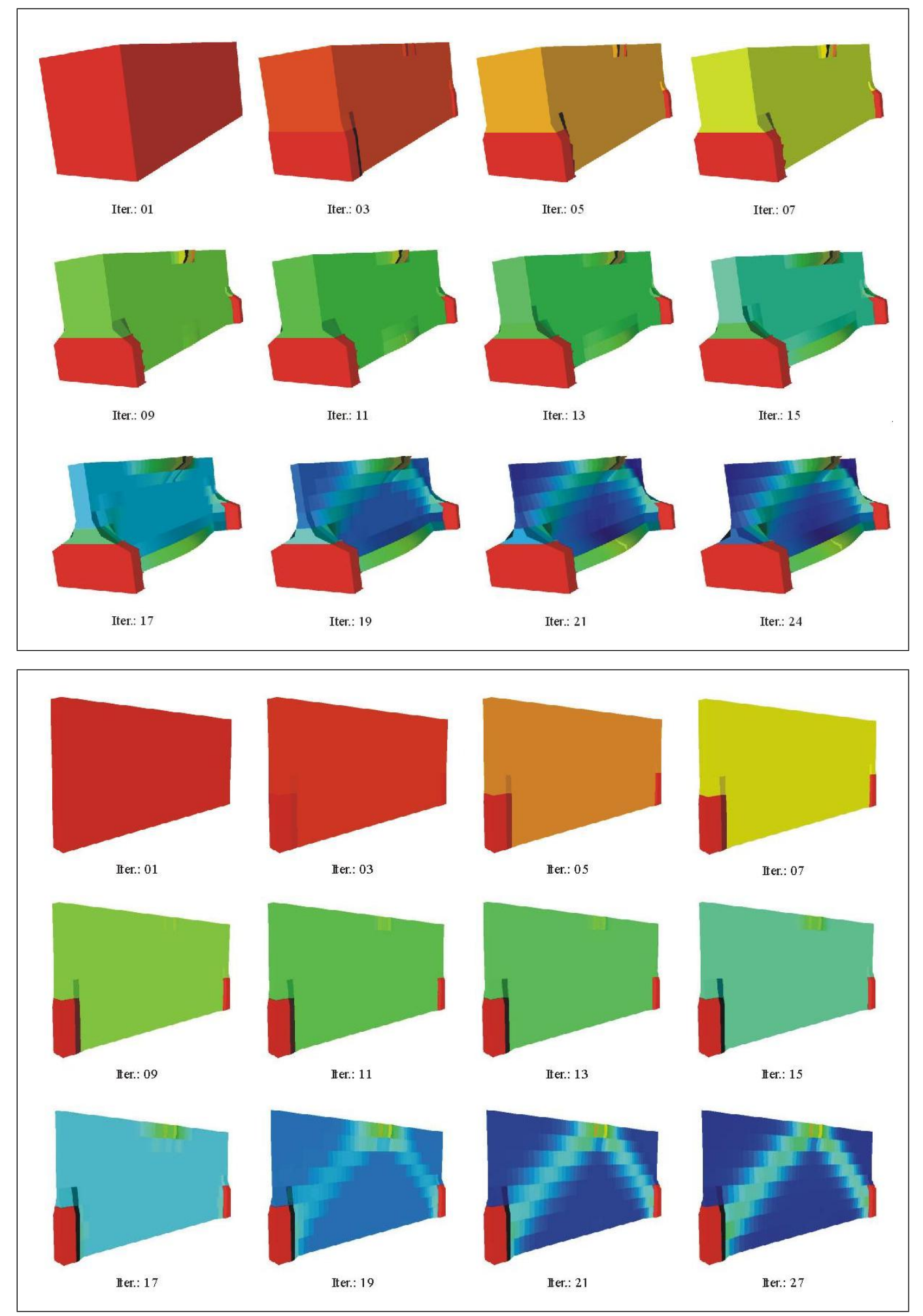

Figure 3. MWSC topology optimization of a simply supported structure, with large height/length ratio, considering sliding (up) and fixed (down) supports. Concentrated load applied in the center of the upper side. (Notes: the supports are not optimized; the entire hollowing out is not allowed.) 


\section{Application Examples}

The examples presented below are bidimensional, the width of the structures is constant, and we perform a plane stress analysis. However, the results are represented as 3-D images [9], being the false width proportional to the relative density of each element.

Figures 2 and 3 show the results for a simply supported structure, with small and large height/length ratio respectively, both for sliding and fixed supports. The domain containing the structure is a prism that bears a concentrated $9000 \mathrm{KN}$ load (vertical, downwards) in the center of the upper side. We analyze half of the structure, because of symmetry. The supports are not optimized. The domain is discretized in 24 times 8 elements (8-node quadrilateral). The material density is $\gamma_{\text {mat }}=7650 \mathrm{Kg} / \mathrm{m}^{3}$.

Type (18) constraints

$$
\sigma_{v m}-\sigma_{e} \leq 0, \quad \sigma^{I} 2 \sigma_{e} \leq 0 \quad \text { and } \quad-2 \sigma_{e}-\sigma^{I I I} \leq 0,
$$

are imposed at the center of all the elements in terms of the elastic stress limit $\sigma_{e}$, being

$$
\sigma_{v m}=\sqrt{\frac{1}{2}\left[\left(\sigma^{I}-\sigma^{I I}\right)^{2}+\left(\sigma^{I I}-\sigma^{I I I}\right)^{2}+\left(\sigma^{I I}-\sigma^{I}\right)^{2}\right]}
$$

the Von Mises reference stress. The absolute value of the stress is limited to the double of the elastic stress limit, in accordance with the standard NBE EA-95 [16].

In figure 2 the domain is $32 \mathrm{~m}$ long, $1.5 \mathrm{~m}$ high and $1 \mathrm{~m}$ wide, and the material is steel with elastic stress limit $\sigma_{e}=230000 \mathrm{KN} / \mathrm{m}^{2}$. We notice that the result obtained in the first case is a clear double $\mathrm{T}$ shaped beam with variable section. The width of the wings increases from the supports to the center of the span, where the load is applied. The result obtained in the second case is similar. However, the central section is closer to a T shaped beam. Actually, the lower wing nearly disappears, since the tension due to the bending is balanced with the compression due to the fixed supports.

In figure 3 the domain is $32 \mathrm{~m}$ long, $12 \mathrm{~m}$ high and $1 \mathrm{~m}$ wide, and the material is fictitious with elastic stress limit $\sigma_{e}=8000 \mathrm{KN} / \mathrm{m}^{2}$. We notice that the result obtained in the first case is clearly a cable stayed arch. The result obtained in the second case is an arch too, but the tie looses its raison d'tre and it disappears, since the supports are fixed. 


\section{Conclusions}

In this paper we present a minimum weight with stress constraints (MWSC) approach for topology structural optimization problems.

The formulation is derived by introducing minimal modifications to a FEM model for linear elasticity problems with small displacements and small displacement gradients.

Although the objective function is simple, as a general rule, this approach leads to more complicated optimization problems with more computational requirements than the maximum stiffness formulations, since a large number of highly non-linear constraints must be taken into account to limit the maximum allowable displacement and stress.

In return, the physical meaning of the optimization statement is closer to the engineering point of view, while any kind of constraint can be included and multiple load cases can be considered.

The formulation has been implemented in a topology optimization system, and several application examples have been solved. The experience shows that this approach does not require neither stabilization nor penalty techniques to produce acceptable results.

The optimized solutions seem to be correct from the engineering point of view and their appearence could be considered closer to the engineering intuition than the traditional truss-like results obtained by the maximum stiffness approach.

\section{Acknowledgements}

This work has been partially supported by Grant Numbers DPI2002-00297 and DPI20010556 of the "Ministerio de Ciencia y Tecnología" of the Spanish Government, by Grant Numbers PGIDIT03-PXIC118001PN and PGIDIT03PXIC118002PN of the DXID-CIIC of the "Xunta de Galicia", and by research fellowships of the "Universidad de A Coruña" and the "Fundación de la Ingeniería Civil de Galicia”. 


\section{References}

[1] Schmidt L.A. Structural design by systematic synthesis. Proc. of the Second ASCE Conference on Electronic Computation, Pittsburgh: ASCE, 1960; 105122.

[2] Hernández S. Métodos de Diseño Óptimo de Estructuras. Madrid: Colegio de Ingenieros de Caminos, Canales y Puertos, 1990.

[3] Navarrina F., Casteleiro M. A general methodologycal analysis for optimum design. International Journal of Numerical Methods in Engineering 1991; 31: 85-111.

[4] Navarrina F., López S., Colominas I., Bendito E., Casteleiro M. High order shape design sensitivity: A unified approach. Computer Methods in Applied Mechanics and Engineering 2000; 188: 681-696.

[5] Bendsøe M.P., Kikuchi N. Generating optimal topologies in structural design using a homogenization method. Computer Methods in Applied Mechanics and Engineering 1988; 71: 197-224.

[6] Ramm E., Schwarz S., Kemmler R. Advances in structural optimization including nonlinear mechanics. Proc. of the European Congress on Computational Methods in Applied Sciences and Engineering [ECCOMAS 2000] (CD-ROM, ISBN: 84-89925-70-4), Barcelona: ECCOMAS, 2000.

[7] Bendsøe M.P. Optimization of structural topology, shape, and material. Heidelberg: Springer-Verlag, 1995.

[8] Ramm E., Maute K., Schwarz S. Adaptive topology and shape optimization. Computational Mechanics: New trends and applications, Proc. of the IV-World Conference on Computational Mechanics (CD-ROM), S. Idelshon, E. Oñate \& E. Dvorkin (Eds.), Barcelona: CIMNE, 1998.

[9] Muiños I. Optimización Topológica de Estructuras: Una Formulación de Elementos Finitos para la Minimización del Peso con Restricciones en Tensión. Proyecto Técnico, ETSICCP. A Corua: Universidad de A Coruña, 2001.

[10] Bendsøe M.P. Variable-topology optimization: status and challenges. Proc. of the European Conference on Computational Mechanics [ECCM'99], W. Wunderlich (Ed.), Munich: TUM, 1999.

[11] Muiños I., Colominas I., Navarrina F., Casteleiro M. Una formulación de mínimo peso con restricciones en tensión para la optimización topológica de estructuras. Métodos Numéricos en Ingeniería y Ciencias Aplicadas, E. Oñate, F. Zárate, G. Ayala, S. Botello \& M.A. Moreles (Eds.), Barcelona: CIMNE, 2002; 399-408.

[12] Navarrina F., Muiños I., Colominas I., Casteleiro M. Optimización Topológica de Estructuras: Una formulación de mínimo peso con restricciones en tensión. Métodos Numéricos en Ingeniería V (Libro y CD-ROM, ISBN: 84-95999-03X), J.M. Goicolea, C. Mota Soares, M. Pastor \& G. Bugeda (Eds.), Barcelona: SEMNI, 2002. 
[13] Hughes T.J.R. The Finite Element Method: Linear Static and Dynamic Finite Element Analysis. New York: Dover Publishers, 2000.

[14] Johnson C. Numerical Solution of Partial Differential Equations by the Finite Element Method. New York: Cambridge University Press, 1990.

[15] Navarrina F., Tarrech R., Colominas I., Mosqueira G., Gómez-Calviño J., Casteleiro M. An efficient MP algorithm for structural shape optimization problems. Computer Aided Optimum Design of Structures VII, S. Hernández \& C.A. Brebbia (Eds.), Southampton: WIT Press, 2001; 247-256.

[16] Ministerio de Fomento. NBE EA-95 Estructuras de acero en edificación. Madrid: Centro de Publicaciones del Ministerio de Fomento, 1998. 